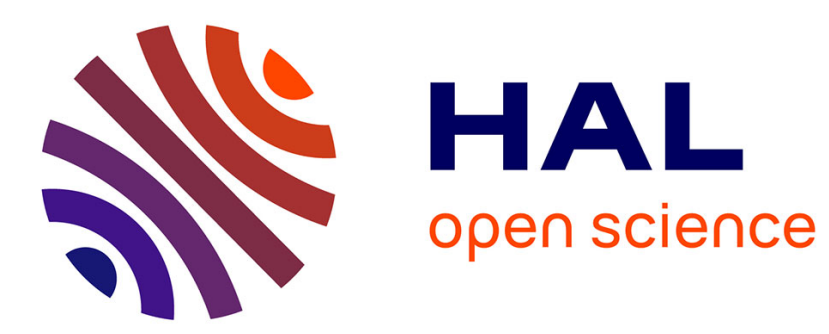

\title{
Stochastic Approximations and Differential Inclusions
} Michel Benaïm, Josef Hofbauer, Sylvain Sorin

\section{To cite this version:}

Michel Benaïm, Josef Hofbauer, Sylvain Sorin. Stochastic Approximations and Differential Inclusions. 2003. hal-00242990

\section{HAL Id: hal-00242990 https://hal.science/hal-00242990}

Preprint submitted on 6 Feb 2008

HAL is a multi-disciplinary open access archive for the deposit and dissemination of scientific research documents, whether they are published or not. The documents may come from teaching and research institutions in France or abroad, or from public or private research centers.
L'archive ouverte pluridisciplinaire HAL, est destinée au dépôt et à la diffusion de documents scientifiques de niveau recherche, publiés ou non, émanant des établissements d'enseignement et de recherche français ou étrangers, des laboratoires publics ou privés. 


\title{
ECOLE POLYTECHNIQUE
}

CENTRE NATIONAL DE LA RECHERCHE SCIENTIFIQUE

Stochastic Approximations and Differential Inclusions

\author{
Michel Benaim \\ Josef Hofbauer \\ Sylvain Sorin
}

December 2003

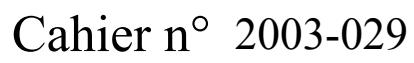

\section{LABORATOIRE D'ECONOMETRIE}

1rue Descartes F-75005 Paris

(33) 155558215

http://ceco.polytechnique.fr/

mailto:labecox@poly.polytechnique.fr 


\title{
Stochastic Approximations and Differential Inclusions
}

\author{
Michel Benaim \\ Josef Hofbauer \\ Sylvain Sorin
}

December 2003

Cahier $n^{\circ}$ 2003-029

Résumé: L'approche en termes de systèmes dynamiques de l'approximation stochastique est étendue au cas ou l'équation différentielle moyenne est remplacée par une inclusion différentielle.

Le théorème de Benaim et Hirsch sur l'ensemble limite est étendu a ce cas. On étudie en détail les ensembles ICT et les attracteurs. On donne des applications a des questions de théorie des jeux, en particulier concernant le théorème d'approchabilite de Blackwell et la convergence de "fictitious play".

\begin{abstract}
The dynamical systems approach to stochastic approximation is generalized to the case where the mean differential equation is replaced by a differential inclusion.

The limit set theorem of Benal" $\{\mathrm{i}\} \mathrm{m}$ and Hirsch is extended to this situation. Internally chain transitive sets and attractors are studied in detail for set-valued dynamical systems. Applications to game theory are given, in particular to Blackwell's approachability theorem and the convergence of fictitious play.
\end{abstract}

Mots clés : $\quad$ Approximation stochastique, système dynamique multivalue

Key Words : Stochastic approximation, multivalued dynamical systems

Classification AMS: $91 \mathrm{~A} 05,37 \mathrm{C}$ 


\title{
Stochastic Approximations and Differential Inclusions *
}

\author{
Michel Benaïm \\ Institut de Mathematiques \\ Université de Neuchâtel, \\ Rue Emile-Argand 11, Suisse \\ michel.benaim@unine.ch \\ Josef Hofbauer ${ }^{\dagger}$ \\ Institut für Mathematik, Universität Wien \\ Strudlhofgasse 4, 1090 Wien, Austria \\ josef.hofbauer@univie.ac.at \\ Sylvain Sorin \\ Laboratoire d'Econométrie, Ecole Polytechnique, \\ 1 rue Descartes, 75005 Paris \\ and Equipe Combinatoire et Optimisation, UFR 921, \\ Université P. et M. Curie - Paris 6, \\ 175 Rue du Chevaleret, 75013 Paris, France \\ sorin@poly.polytechnique.fr
}

25 Nov 03

${ }^{*}$ This research was started during visits of JoH in Paris in 2002. JoH thanks the Laboratoire d'Econométrie, Ecole Polytechnique and the D.E.A. OJME, Université P. et M. Curie Paris 6, for financial support and SyS for his hospitality. MiB thanks the Erwin Schrödinger Institute. JoH also thanks the Austrian Science Fund P15281.

†address after 1 Jan 2004: Department of Mathematics, University College London, London WC1E 6BT, U.K. 


\begin{abstract}
The dynamical systems approach to stochastic approximation is generalized to the case where the mean differential equation is replaced by a differential inclusion. The limit set theorem of Benaïm and Hirsch is extended to this situation. Internally chain transitive sets and attractors are studied in detail for set-valued dynamical systems. Applications to game theory are given, in particular to Blackwell's approachability theorem and the convergence of fictitious play.
\end{abstract}

\title{
1 Introduction
}

\subsection{Presentation}

A powerful method for analyzing stochastic approximations or recursive stochastic algorithms is the so-called "ODE (Ordinary Differential Equation) method" which allow to describe the limit behavior of the algorithm in terms of the asymptotics of a certain ODE

$$
\frac{d x}{d t}=F(x)
$$

obtained by suitable averaging.

This method was introduced by Ljung (1977) and extensively studied thereafter (see e.g the books by Kushner and Yin (1997) or Duflo (1996) for a comprehensive introduction and further references). However until recently most works in this direction have assumed the simplest dynamics for $F$. For example that $F$ is linear or given by the gradient of a cost function. While this type of assumption makes perfect sense in engineering applications (where algorithms are often designed to minimize a cost function) there are several situations, including models of learning or adaptive behavior in games, for which $F$ may have more complicated dynamics.

In a series of papers Benaïm $(1996,1999)$ and Benaïm and Hirsch (1996b) have demonstrated that the asymptotic behavior of stochastic approximation processes can be described with great deal of generality beyond gradients and other simple dynamics. One of the key results is that the limit sets of the process are almost surely compact connected attractor free (or internally chain transitive sets in the sense of Conley (1978)) for the deterministic flow induced by $F$.

The purpose of this paper is to show that such a dynamical system approach easily extends to the situation where the mean ODE is replaced by a differential 
inclusion. This is strongly motivated by certain problems arising in economics and game theory. In particular, the results here allow to give a simple and unified presentation to Blackwell's approachability theorem, Smale's results on prisoner's dilemma, as well as convergence of fictitious play in potential games. Many others applications will be considered in a forthcoming paper, Benaim, Hofbauer and Sorin (2003), the present one being mainly devoted to theoretical issues.

The organization of the paper is as follows. Part 1 introduces the different notions of solutions, perturbed solutions and stochastic approximations associated to a differential inclusion. Part 2 is devoted to the presentation of two classes of examples. Part 3 is a general study of the dynamical system defined by a differential inclusion. The main result (Theorem 3.5) on the limit set of a perturbed solution being internally chain transitive is stated . Then related notions: invariant and attracting sets, attractors and Lyapounov functions are analyzed. Part 4 countains the proof of the limit set Theorem. Finally Part 5 applies the previous results to two adaptive processes in Game Theory: approachability and fictitious play.

\subsection{The differential inclusion}

Let $F$ denote a set-valued function mapping each point $x \in \mathbb{R}^{m}$ to a set $F(x) \subset$ $\mathbb{R}^{m}$. We suppose throughout that

Hypothesis 1.1 (Standing assumptions on F).

(i) $F$ is a closed set-valued map. That is

$$
\operatorname{Graph}(F)=\{(x, y): y \in F(x)\}
$$

is a closed subset of $\mathbb{R}^{m} \times \mathbb{R}^{m}$.

(ii) $F(x)$ is a nonempty compact convex subset of $\mathbb{R}^{m}$ for all $x \in \mathbb{R}^{m}$.

(iii) There exists $c>0$ such that for all $x \in \mathbb{R}^{m}$

$$
\sup _{z \in F(x)}\|z\| \leq c(1+\|x\|)
$$

where $\|\cdot\|$ denotes any norm on $\mathbb{R}^{m}$.

Definition (I) A solution for the differential inclusion

$$
\frac{d \mathbf{x}}{d t} \in F(\mathbf{x})
$$


with initial point $x \in \mathbb{R}^{m}$ is an absolutely continuous mapping $\mathbf{x}: \mathbb{R} \rightarrow \mathbb{R}^{m}$ such that $\mathbf{x}(0)=x$ and

for almost every $t \in \mathbb{R}$.

$$
\frac{d \mathbf{x}(t)}{d t} \in F(\mathbf{x}(t))
$$

Under the above assumptions, it is well-known (see Aubin and Celina (1984), Ch. 4.2, or Clarke et al (1998), Ch. 4.1) that (I) admits (typically non-unique) solutions through every initial point.

Remark 1.2 Suppose a differential inclusion is given on a compact convex set $C \subset \mathbb{R}^{m}$, of the form $F(x)=\Phi(x)-x$, such that $\Phi(x) \subset C$ for all $x \in C$ and $\Phi$ satisfies (i) and (ii), with $\mathbb{R}^{m}$ replaced by $C$. Then we can extend it to a differential inclusion defined on the whole space $\mathbb{R}^{m}$ : For $x \in \mathbb{R}^{m}$ let $P(x) \in C$ denote the unique point in $C$ closest to $x$ and define $F(x)=\Phi(P(x))-x$. Then $F$ satisfies Hypothesis 1.1.

\subsection{Perturbed solutions}

The main object of this paper are paths which are obtained as certain (deterministic or random) perturbations of solutions of $(I)$.

Definition (II) A continuous function $\mathbf{y}: \mathbb{R}_{+}=[0, \infty) \rightarrow \mathbb{R}^{m}$ will be called a perturbed solution to $(I)$ (we also say a perturbed solution to $F$ ) if it satisfies the following set of conditions $(I I)$ :

(i) $\mathbf{y}$ is absolutely continuous,

(ii) $\frac{d \mathbf{y}(t)}{d t}-U(t) \in F^{\delta(t)}(\mathbf{y}(t))$ for almost every $t>0$, for some function $\delta$ : $[0, \infty) \rightarrow \mathbb{R}$ with $\delta(t) \rightarrow 0$ as $t \rightarrow \infty$. Here $F^{\delta}(x):=\left\{y \in \mathbb{R}^{m}: \exists z:\right.$ $\|z-x\|<\delta, d(y, F(z))<\delta\}$ and $d(y, C)=\inf _{c \in C}\|y-c\|$.

(iii) $t \mapsto U(t)$ is a locally integrable function such that

$$
\lim _{t \rightarrow \infty} \sup _{0 \leq v \leq T}\left\|\int_{t}^{t+v} U(s) d s\right\|=0
$$

for all $T>0$.

The purpose of this paper is to investigate the long term behavior of $\mathbf{y}$ and to describe its limit set

$$
L(\mathbf{y})=\bigcap_{t \geq 0} \overline{\{\mathbf{y}(s): s \geq t\}}
$$

in terms of the dynamics induced by $F$. 


\subsection{Stochastic approximations}

As it will be shown here, a natural class of perturbed solutions to $F$ arise from certain stochastic approximation processes.

Definition (III) A discrete time process $\left\{x_{n}\right\}_{n \in \mathbb{N}}$ living in $\mathbb{R}^{m}$ is a solution of (III) if it verifies a recursion of the form

$$
x_{n+1}-x_{n}-\gamma_{n+1} U_{n+1} \in \gamma_{n+1} F\left(x_{n}\right)
$$

where the characteristics $\gamma$ and $U$ satisfy

- $\left\{\gamma_{n}\right\}_{n \geq 1}$ is a sequence of nonnegative numbers such that

$$
\sum_{n} \gamma_{n}=\infty, \lim _{n \rightarrow \infty} \gamma_{n}=0
$$

- $U_{n} \in \mathbb{R}^{m}$ are (deterministic or random) perturbations.

To such a process is naturally associated a continuous time process as follows. Definition (IV) Set

$$
\tau_{0}=0 \text { and } \tau_{n}=\sum_{i=1}^{n} \gamma_{i} \text { for } n \geq 1
$$

and define the continuous time affine interpolated process $\mathbf{w}: \mathbb{R}_{+} \rightarrow \mathbb{R}^{m}$ by

$$
\mathbf{w}\left(\tau_{n}+s\right)=x_{n}+s \frac{x_{n+1}-x_{n}}{\tau_{n+1}-\tau_{n}}, \quad s \in\left[0, \gamma_{n+1}\right)
$$

\subsection{From interpolated process to perturbed solutions}

The next result give sufficient conditions on the characteristics of the discrete process $(I I I)$ for its interpolation $(I V)$ to be a perturbed solution $(I I)$.

Proposition 1.3 Assume that

(i) For all $T>0$

$$
\lim _{n \rightarrow \infty} \sup \left\{\left\|\sum_{i=n}^{k-1} \gamma_{i+1} U_{i+1}\right\|: k=n+1, \ldots, m\left(\tau_{n}+T\right)\right\}=0
$$

where

$$
m(t)=\sup \left\{k \geq 0: t \geq \tau_{k}\right\}
$$


(ii) $\sup _{n}\left\|x_{n}\right\|=M<\infty$,

then the interpolated process $\mathbf{w}$ is a perturbed solution of $F$.

Proof Let $\mathbf{U}, \gamma: \mathbb{R}_{+} \rightarrow \mathbb{R}^{m}$ denote the continuous time processes defined by

$$
\mathbf{U}\left(\tau_{n}+s\right)=U_{n+1}, \quad \gamma\left(\tau_{n}+s\right)=\gamma_{n+1}
$$

for all $n \in \mathbb{N}, 0 \leq s<\gamma_{n+1}$.

Then, for $t \in\left[\tau_{n}, \tau_{n+1}\right)$ :

$$
\mathbf{w}(t) \in x_{m(t)}+(t-m(t))\left[\mathbf{U}(t)+F\left(x_{m(t)}\right)\right]
$$

hence

$$
\dot{\mathbf{w}}(t) \in \mathbf{U}(t)+F\left(x_{m(t)}\right) .
$$

Let us put $\delta(t)=\left\|\mathbf{w}(t)-x_{m(t)}\right\|$. Then obviously

$$
F\left(x_{m(t)}\right) \subset F^{\delta(t)}(\mathbf{w}(t)) .
$$

In addition:

$$
\delta(t) \leq \gamma_{m(t)+1}\left[\left\|U_{m(t)+1}\right\|+c(1+M)\right]
$$

hence goes to 0 , using hypothesis (i) of the statement of the Proposition. It remains to check condition (iii) of $(I I)$ but one has

$$
\begin{aligned}
\left\|\int_{t}^{t+v} \mathbf{U}(s) d s\right\| \leq & \gamma_{m(t)+1}\left\|U_{m(t)+1}\right\|+\left\|\sum_{\ell=m(t)+1}^{m(t+v)-1} \gamma_{\ell+1} U_{\ell+1}\right\| \\
& +\gamma_{m(t+v)+1}\left\|U_{m(t+v)+1}\right\|
\end{aligned}
$$

and the result follows from condition (i). QED

\subsection{Sufficient conditions}

Let $(\Omega, \mathcal{F}, P)$ be a probability space and $\left\{\mathcal{F}_{n}\right\}_{n \geq 0}$ a filtration of $\mathcal{F}$ (i.e., a nondecreasing sequence of sub- $\sigma$-algebras of $\mathcal{F}$ ). We say that a stochastic process $\left\{x_{n}\right\}$ given by $(I I I)$ satisfies the Robbins-Monro or Martingale difference noise (Kushner and Yin, 1997) condition if its characteristics satisfy:

(i) $\left\{\gamma_{n}\right\}$ is a deterministic sequence. 
(ii) $\left\{U_{n}\right\}$ is adapted to $\left\{\mathcal{F}_{n}\right\}$. That is, $U_{n}$ is measurable with respect to $\mathcal{F}_{n}$ for each $n \geq 0$.

(iii) $\mathrm{E}\left(U_{n+1} \mid \mathcal{F}_{n}\right)=0$.

The next proposition is a classical estimate for stochastic approximation processes. Note that $F$ does not appear. We refer the reader to (Benaïm, 1999, Propositions 4.2 and 4.4) for a proof and further references.

Proposition 1.4 Let $\left\{x_{n}\right\}$ given by $(I I I)$ be a Robbins-Monro process. Suppose that one of the following condition holds

(i) For some $q \geq 2$

$$
\sup _{n} \mathrm{E}\left(\left\|U_{n}\right\|^{q}\right)<\infty
$$

and

$$
\sum_{n} \gamma_{n}^{1+q / 2}<\infty
$$

or

(ii) There exists a positive number $\Gamma$ such that for all $\theta \in \mathbb{R}^{m}$

$$
\mathrm{E}\left(\exp \left(\left\langle\theta, U_{n+1}\right\rangle\right) \mid \mathcal{F}_{n}\right) \leq \exp \left(\frac{\Gamma}{2}\|\theta\|^{2}\right)
$$

and

$$
\sum_{n} e^{-c / \gamma_{n}}<\infty
$$

for each $c>0$.

Then assumption (i) of Proposition 1.3 holds with probability 1.

Remark 1.5 Typical applications are:

(i) $U_{n}$ uniformly bounded in $L^{2}$ and $\gamma_{n}=1 / n$,

(ii) $U_{n}$ uniformly bounded and $\gamma_{n}=o\left(\frac{1}{\log (n)}\right)$. 


\section{Examples}

\subsection{A multistage decision making model}

Let $A$ and $B$ be measurable spaces respectively called the action space and the states of nature, $E \subset \mathbb{R}^{m}$ a convex compact set called the outcomes space and $H: A \times B \rightarrow E$ a measurable function called the outcome function.

At discrete times $n=1,2 \ldots$ a decision maker (DM) chooses an action $a_{n}$ from $A$ and observes an outcome $H\left(a_{n}, b_{n}\right)$. We suppose that

(i) The sequence $\left\{a_{n}, b_{n}\right\}_{n \geq 0}$ is a random process defined on some probability space $(\Omega, \mathcal{F}, P)$ and adapted to some filtration $\left\{\mathcal{F}_{n}\right\}$. Here $\mathcal{F}_{n}$ has to be understood as the history of the process until time $n$.

(ii) Given the history $\mathcal{F}_{n}, \mathrm{DM}$ and nature act independently:

$$
\mathrm{P}\left(\left(a_{n+1}, b_{n+1}\right) \in d a \times d b \mid \mathcal{F}_{n}\right)=\mathrm{P}\left(a_{n+1} \in d a \mid \mathcal{F}_{n}\right) \mathrm{P}\left(b_{n+1} \in d b \mid \mathcal{F}_{n}\right)
$$

for any measurable sets $d a \subset A$ and $d b \subset B$.

(iii) DM only keeps track on the cumulative average of the past outcomes:

$$
x_{n}=\frac{1}{n} \sum_{i=1}^{n} H\left(a_{i}, b_{i}\right)
$$

and his decisions are based on this average. That is

$$
\mathrm{P}\left(a_{n+1} \in d a \mid \mathcal{F}_{n}\right)=Q_{x_{n}}(d a)
$$

where $Q_{x}($.$) is a probability measure over A$ for each $x \in E$, and $x \in E \mapsto$ $Q_{x}(d a) \in[0,1]$ is measurable for each measurable set $d a \subset A$. The family $Q=\left\{Q_{x}\right\}_{x \in E}$ is called a strategy for DM.

This latter assumption can be justified by considerations of limited memory and bounded rationality. It is partially motivated by Smale's approach to the prisoner's dilemma (Smale, 1980) (see also Benaïm and Hirsch, 1996), Blackwell's approachability theory (Blackwell, 1956; Sorin 2002), as well as fictitious play (Brown, 1951; Robinson, 1951) and stochastic fictitious play (Benaïm and Hirsch, 1999; Fudenberg and Levine, 1998; Hofbauer and Sandholm, 2002) in game theory (see the examples below).

For each $x \in E$ let

$$
C(x)=\left\{\int_{A \times B} H(a, b) Q_{x}(d a) \nu(d b): \nu \in \mathcal{P}(B)\right\}
$$


where $\mathcal{P}(B)$ denotes the set of probability measures over $B$. Then clearly

$$
\mathrm{E}\left(H\left(a_{n+1}, b_{n+1}\right) \mid \mathcal{F}_{n}\right) \in C\left(x_{n}\right) \subset \bar{C}(x)
$$

where $\bar{C}$ denote the smallest closed set-valued extension of $C$ with convex values. More precisely, the graph of $\bar{C}$ is the intersection of all closed subsets $G \subset E \times E$ for which the fiber $G_{x}=\{y \in E:(x, y) \in G\}$ is convex and contains $C(x)$.

For $x \in \mathbb{R}^{m}$ let $P(x)$ denote the unique point in $E$ closest to $x$. Extend $\bar{C}$ as in Remark 1.2 to a set-valued map on $\mathbb{R}^{m}$ by setting

$$
\widehat{C}(x)=\bar{C}(P(x)) .
$$

Then the map

$$
F(x)=-x+\bar{C}(P(x))=-x+\widehat{C}(x)
$$

clearly satisfies Hypothesis 1.1 and $\left\{x_{n}\right\}$ verifies the recursion

$$
x_{n+1}-x_{n}=\frac{1}{n+1}\left(-x_{n}+H\left(a_{n+1}, b_{n+1}\right)\right)
$$

which can be rewritten as $(I I I)$

$$
x_{n+1}-x_{n} \in \gamma_{n+1}\left[F\left(x_{n}\right)+U_{n+1}\right]
$$

with $\gamma_{n}=\frac{1}{n}$ and $U_{n+1}=H\left(a_{n+1}, b_{n+1}\right)-\int_{A} H\left(a, b_{n+1}\right) Q_{x_{n}}(d a)$. Hence, the conditions of Proposition 1.4 are satisfied and one deduces

Proposition 2.1 The affine continuous time interpolated process (IV) of the process $\left\{x_{n}\right\}$ given by (2) is almost surely a perturbed solution of $F$ defined by (3).

Example 2.2 (Blackwell's approachability theory). A set $\Lambda \subset E$ is said approachable if there exists a strategy $Q$ such that $x_{n} \rightarrow \Lambda$ almost surely. Blackwell (1956) give conditions ensuring approachability. We will show in section 5.1 how Blackwell's results can be partially derived from our main results and generalized (Corollary 5.2) in certain directions.

\subsection{Learning in Games}

The preceeding formalism is well suited to analyze certain models of learning in games. 
Consider the situation where $m$ players are playing a game over and over. Let $A^{i}$ (for $i \in I=\{1, \ldots, m\}$ ) be a finite set representing the actions (pure strategies) available to player $i$; and let $X^{i}$ be the finite dimensional simplex of probabilities over $A^{i}$ (the set of mixed strategies for player $i$.). For $i \in I$ we let $A^{-i}$ and $X^{-i}$ respectively denote the actions and mixed strategies available to the opponents of $i$. The payoff function to player $i$ is given by a function $U^{i}: A^{i} \times A^{-i} \rightarrow \mathbb{R}$. As usual we extend $U^{i}$ to a function (still denoted $U^{i}$ ) on $X^{i} \times X^{-i}$, by multi-linearity.

Example 2.3 (Fictitious and stochastic fictitious play). Consider the game from the viewpoint of player $i$ so that the decision maker is player $i$ and "nature" is given by the other players. In fictitious or stochastic fictitious play the outcome space is the space $X^{i} \times X^{-i}$ of mixed strategies and the outcome function is the "identity" function $H: A^{i} \times A^{-i} \rightarrow X^{i} \times X^{-i}$ mapping every profile of actions $a$ to the corresponding profile of mixed strategy $\delta_{a}$.

Let

$$
B R^{i}\left(x^{-i}\right)=\underset{a^{i} \in A^{i}}{\operatorname{Argmax}} U^{i}\left(a^{i}, x^{-i}\right) \subset A^{i}
$$

be the set of best actions that $i$ can play in response to $x^{-i}$.

Both classical fictitious play (Brown, 1951, Robinson 1951) and stochastic fictitious play (Benaïm and Hirsch 1999, Fudenberg and Levine 1998, Hofbauer and Sandholm 2002) assume that the strategy of player $i, Q^{i}=\left\{Q_{x}^{i}\right\}$, can be written as

$$
Q_{x}^{i}\left(a^{i}\right)=q^{i}\left(a^{i}, x^{-i}\right)
$$

where $q^{i}: A^{i} \times X^{-i} \rightarrow[0,1]$ is such that

\section{Fictitious Play Assumption :}

$$
\sum_{a^{i} \in B R^{i}\left(x^{-i}\right)} q^{i}\left(a^{i}, x^{-i}\right)=1 ;
$$

or

Stochastic Fictitious Play Assumption : $q^{i}$ is smooth in $x^{-i}$ and

$$
\sum_{a^{i} \in B R^{i}\left(x^{-i}\right)} q^{i}\left(a^{i}, x^{-i}\right) \geq 1-\delta
$$

for some $0<\delta<<1$.

In this framework, if $a_{\ell}$ denotes the profile of actions at stage $\ell$, one has

$$
x_{n}=\frac{1}{n} \sum_{\ell=1}^{n} a_{\ell}
$$


and

$$
x_{n+1}-x_{n}=\frac{1}{n+1}\left(a_{n+1}-x_{n}\right)
$$

Thus for each $i$

$$
x_{n+1}^{i}-x_{n}^{i} \in \frac{1}{n+1}\left(\overline{B R}^{i}\left(x_{n}^{-i}\right)-x_{n}^{i}\right)
$$

where $\overline{B R}^{i}\left(x^{-i}\right) \subset X^{i}$ is the convex hull of $B R^{i}\left(x^{-i}\right)$ for the standard fictitious play; and $\overline{B R}^{i}(x)=\sum_{a^{i} \in A^{i}} q^{i}\left(a^{i}, x^{-i}\right) \delta_{a^{i}}$ for the stochastic fictitious play.

Thus the set-valued map $F$ defined in (3) is given as

$$
F^{i}(x)=-x+\overline{B R}^{i}\left(x^{-i}\right) \times X^{-i}
$$

Observe that if a subset $J \subset I$ of players plays a fictitious (or stochastic fictitious) play strategy, then $F^{i}$ has to be replaced by

$$
F^{J}(x)=\bigcap_{i \in J} F^{i}(x) .
$$

In particular, if all players play a fictitious play strategy, the differential inclusion induced by $F$ is the best response differential inclusion (Gilboa and Matsui (1991), Hofbauer (1995), Hofbauer and Sorin (2002)); while if all play a stochastic fictitious play, $F$ is a smooth best response vector field (Benaïm and Hirsch (1999), Fudenberg and Levine (1998), Hofbauer and Sandholm (2002)).

Example 2.4 (Smale approach to prisoner's dilemma). We still consider the game from the viewpoint of player $i$ so that DM is player $i$ and nature the other players, but we take for $H$ the payoff vectors function:

$$
\begin{gathered}
H: A^{i} \times A^{-i} \rightarrow E, \\
a \rightarrow U(a)=\left(U^{1}(a), \ldots, U^{m}(a)\right) ;
\end{gathered}
$$

where $E \subset \mathbb{R}^{m}$ is the convex hull of the payoff vectors $\{U(a)\}$.

This setting fits exactly with Smale's approach to prisoner's Dilemma (Smale, 1980) later revisited by Benaïm and Hirsch (1996a). Details will be given in section 5.2 where Smale's approach will be reinterpreted in the framework of approachability. 


\section{Set valued dynamical systems}

\subsection{Properties of the trajectories of $(I)$}

Let $C^{0}\left(\mathbb{R}, \mathbb{R}^{m}\right)$ denote the space of continuous paths $\left\{\mathbf{z}: \mathbb{R} \rightarrow \mathbb{R}^{m}\right\}$ equipped with the topology of uniform convergence on compact intervals. This is a complete metric space for the distance $\mathbf{D}$ defined by

$$
\mathbf{D}(\mathbf{x}, \mathbf{z})=\sum_{k=1}^{\infty} \frac{1}{2^{k}} \min \left(\|\mathbf{x}-\mathbf{z}\|_{[-k, k]}, 1\right),
$$

where $\|\cdot\|_{[-k, k]}$ stands for the supremum norm on $C^{0}\left([-k, k], \mathbb{R}^{m}\right)$.

Given a set $M \subset \mathbb{R}^{m}$ we let $S_{M} \subset C^{0}\left(\mathbb{R}, \mathbb{R}^{m}\right)$ denote the set of all solutions to $(I)$ with initial conditions $x \in M\left(S_{M}=\bigcup_{x \in M} S_{x}\right)$ and $S_{M, M} \subset S_{M}$ the subset consisting of solutions $\mathbf{x}$ that remain in $M$ (i.e., $\mathbf{x}(\mathbb{R}) \subset M$ ).

Lemma 3.1 Assume $M$ compact. Then $S_{M}$ is a nonempty compact set and $S_{M, M}$ is a compact (possibly empty) set.

Proof The first assertion follows from Aubin and Cellina (1984), section 2.2, Thm. 1, p. 104. The second easily follows from the first one. QED

\subsection{Set-valued dynamical system induced by $(I)$.}

The differential inclusion $(I)$ induces a set-valued dynamical system $\left\{\Phi_{t}\right\}_{t \in \mathbb{R}}$ defined by

$$
\Phi_{t}(x)=\{\mathbf{x}(t): \mathbf{x} \text { is a solution to }(I) \text { with } \mathbf{x}(0)=x\} .
$$

The family $\Phi=\left\{\Phi_{t}\right\}_{t \in \mathbb{R}}$ enjoys the following properties:

(a) $\Phi_{0}(x)=\{x\}$,

(b) $\Phi_{t}\left(\Phi_{s}(x)\right)=\Phi_{t+s}(x)$ for all $t, s \geq 0$,

(c) $y \in \Phi_{t}(x) \Rightarrow x \in \Phi_{-t}(y)$ for all $x, y \in \mathbb{R}^{m}, t \in \mathbb{R}$.

(d) $(x, t) \mapsto \Phi_{t}(x)$ is a closed set-valued map with compact values.

Properties $(a),(b),(c)$ are immediate to verify and property $(d)$ easily follows from Lemma 3.1.

For subsets $T \subset \mathbb{R}$ and $A \subset \mathbb{R}^{m}$ we will denote

$$
\Phi_{T}(A)=\bigcup_{t \in T} \bigcup_{x \in A} \Phi_{t}(x)
$$




\section{Invariant sets}

A set $A \subset \mathbb{R}^{m}$ is said to be:

strongly-invariant (for $\Phi$ ) if $A=\Phi_{t}(A)$ for all $t \in \mathbb{R}$;

quasi-invariant if $A \subset \Phi_{t}(A)$ for all $t \in \mathbb{R}$;

semi-invariant if $\Phi_{t}(A) \subset A$ for all $t \in \mathbb{R}$.

invariant (for $F$ ) if for all $x \in A$ there exists a solution $\mathbf{x}$ to $(I)$ with $\mathbf{x}(0)=x$ and such that $\mathbf{x}(\mathbb{R}) \subset A$.

We call a set A strongly positive invariant if $\phi_{t}(A) \subset A$ for all $t>0$.

At first glance (at least for the one used to ordinary differential equations) the good notion might seem to be the one defined by strong invariance. However this notion is too strong for differential inclusions as shown by the simple example below (Example 3.2), and the main notions that will be really needed here are invariance and strong positive invariance. We have included the definition of quasi-invariance mainly because some of our later results may be related to a paper by Bronstein and Kopanskii (1988) making use of this notion ${ }^{1}$. Observe however, that by Lemma 3.3 below, quasi-invariance coincides with invariance for compact sets.

Example 3.2 (a) Let $F$ be the set-valued map defined on $\mathbb{R}$ by $F(x)=$ $-\operatorname{sgn}(x)$ if $x \neq 0$ and $F(0)=[-1,1]$. Then $\Phi_{t}(\{0\})=\{0\}$ for $t \geq 0$ and $\Phi_{t}(\{0\})=[t,-t]$ for $t<0$. Hence $\{0\}$ is invariant and strongly positively invariant but is not strongly invariant.

(b) Let now $F(x)=x$ for $x<0, F(x)=1$ for $x>0$ and $F(0)=[0,1]$. Then $\Phi_{t}(\{0\})=\{0\}$ for $t \leq 0$ and $\Phi_{t}(\{0\})=[0, t]$ for $t \geq 0$. Hence $\{0\}$ is invariant but not strongly positively invariant.

Lemma 3.3 Every invariant set is quasi-invariant. Every compact quasiinvariant set is invariant.

Proof Suppose $A$ is invariant. Let $x \in A$ and $\mathbf{x}$ a solution to $(I)$ with $\mathbf{x}(0)=x$ and $\mathbf{x}(\mathbb{R}) \subset A$. For all $t \in \mathbb{R}$ we have $x \in \Phi_{t}(\mathbf{x}(-t))$. Hence $A$ is quasi-invariant.

Conversely suppose $A$ is quasi-invariant and compact. Choose $x \in A$ and fix $N \in \mathbb{N}$. Then for every $p \in \mathbb{N}$ there exists, by quasi-invariance and by glueing pieces of solutions together, a solution $\mathbf{x}_{p, N}$ to $(I)$ such that $\mathbf{x}_{p, N}(0)=x$ and for all $q \in\left\{-2^{p}, \ldots, 2^{p}\right\}, \mathbf{x}_{p, N}\left(\frac{q N}{2^{p}}\right) \in A$. By Lemma 3.1, the sequence $\left\{\mathbf{x}_{p, N}\right\}_{p \in \mathbb{N}}$ is relatively compact in $C^{0}\left([-N, N], \mathbb{R}^{m}\right)$. Let $\mathbf{x}_{N}$ be a limit point of this sequence. Then for each dyadic point $t=\frac{q N}{2^{p}}$ where $q \in\left\{-2^{p}, \ldots, 2^{p}\right\}, \mathbf{x}_{N}(t) \in \bar{A}$.

\footnotetext{
${ }^{1}$ Invariant sets in Bronstein and Kopanskii (1988) coincide with what we define here as strongly invariant sets.
} 
Continuity of $\mathbf{x}_{N}$ implies $\mathbf{x}_{N}([-N, N]) \subset \bar{A}$. Now let $\mathbf{x}$ be a limit point of the sequence $\left\{\mathbf{x}_{N}\right\}_{N \in \mathbb{N}}$ in $C^{0}\left(\mathbb{R}, \mathbb{R}^{m}\right)$. Then $\mathbf{x}(\mathbb{R}) \subset \bar{A}$ and $\mathbf{x}$ is solution to $(I)$. QED

Remark 3.4 $A$ invariant together with strong positive invariance implies $\phi_{t}(A)=A$ for $t>0$.

\subsection{Chain-recurrence and the limit set theorem}

Given a set $A \subset \mathbb{R}^{m}$ and $x, y \in A$ we write $x \hookrightarrow_{A} y$ if for every $\varepsilon>0$ and $T>0$ there exists an integer $n \in \mathbb{N}$, solutions $\mathbf{x}_{1}, \ldots \mathbf{x}_{n}$ to $(I)$ and real numbers $t_{1}, t_{2}, \ldots, t_{n}$ greater than $T$ such that

(a) $\mathbf{x}_{i}\left(\left[0, t_{i}\right]\right) \subset A$,

(b) $\left\|\mathbf{x}_{i}\left(t_{i}\right)-\mathbf{x}_{i+1}(0)\right\| \leq \varepsilon$ for all $i=1, \ldots, n-1$,

(c) $\left\|\mathbf{x}_{1}(0)-x\right\| \leq \varepsilon$ and $\left\|\mathbf{x}_{n}\left(t_{n}\right)-y\right\| \leq \varepsilon$.

The sequence $\left(\mathbf{x}_{1}, \ldots \mathbf{x}_{n}\right)$ is called an $(\varepsilon, T)$ chain (in $A$ from $x$ to $y$ ) for $F$.

Definition A set $A \subset \mathbb{R}^{m}$ is said internally chain transitive provided $A$ is compact and $x \hookrightarrow_{A} y$ for all $x, y \in A$.

Lemma 3.5 An internally chain transitive set is invariant.

Proof Let $A$ be such a set and $x \in A$. Let $\left(\mathbf{x}_{1}, \ldots \mathbf{x}_{n}\right)$ be an $(\varepsilon, T)$ chain from $x$ to $x$. Set $\mathbf{y}_{\varepsilon, T}(t)=\mathbf{x}_{1}(t)$ for $0 \leq t \leq T$ and $\mathbf{z}_{\varepsilon, T}(t)=\mathbf{x}_{n}\left(t_{n}+t\right)$, for $-T \leq t \leq 0$. By Lemma 3.1 we can extract from $\left(\mathbf{y}_{1 / p, T}\right)_{p \in \mathbb{N}}$ and $\left(\mathbf{z}_{1 / p, T}\right)_{p \in \mathbb{N}}$ some subsequences converging respectively to $\mathbf{y}_{T}$ and $\mathbf{z}_{T}$ where $\mathbf{y}_{T}$ and $\mathbf{z}_{T}$ are solutions to to $(I), \mathbf{y}_{T}(0)=x=\mathbf{z}_{T}(0), \mathbf{y}_{T}([0, T]) \subset A$ and $\mathbf{z}_{T}([-T, 0]) \subset A$. The map $\mathbf{w}_{T}(t)=\mathbf{y}_{T}(t)$ for $t \geq 0$ and $\mathbf{w}_{T}(t)=\mathbf{z}_{T}(t)$ for $t \leq 0$ is then a solution to $(I)$ with initial condition $x$ and such that $\mathbf{w}_{T}([-T, T]) \subset A$. By Lemma 3.1 again we extract from $\left(\mathbf{w}_{T}\right)_{T \geq 0}$ a subsequence converging to a solution $\mathbf{w}$ whose range lies in $A$ and with initial condition $x$. QED

This notion of recurrence due to Conley (1978) for classical dynamical systems is well suited to the description of the asymptotic behavior of a perturbed solution to $(I)$ as shown by the following theorem. 
Theorem 3.6 Let $\mathbf{y}$ be a bounded perturbed solution to $(I)$. Then, the limit set of $\mathbf{y}$,

$$
L(\mathbf{y})=\bigcap_{t \geq 0} \overline{\{\mathbf{y}(s): s \geq t\}}
$$

is internally chain transitive.

This theorem is the set-valued version of the limit set theorem proved by Benaïm (1996) for stochastic approximation and Benaïm and Hirsch (1996b) for asymptotic pseudo trajectories of a flow. We will deduce it from the more general results of section 4 .

\subsection{Limit sets}

The set

$$
\omega_{\Phi}(x):=\bigcap_{t \geq 0} \overline{\Phi_{[t, \infty)}(x)}
$$

is the $\omega$-limit set of a point $x \in \mathbb{R}^{m}$. Note that $\omega_{\Phi}(x)$ contains the limit sets $L(\mathbf{x})$ of all solutions $\mathbf{x}$ with $\mathbf{x}(0)=x$ but is in general larger than the union of these.

In contrast to the limit set of a solution, the $\omega$-limit set of a point need not be internally chain transitive.

Example 3.7 Let $F$ be the set-valued map defined on $\mathbb{R}$ by $F(x)=1-x$ for $x>0$ and $F(0)=[0,1]$ and $F(x)=-x$ for $x<0$. Then for every solution $\mathbf{x}$, one has $\lim _{t \rightarrow \infty} \mathbf{x}(t)=0$ or 1 . But $\omega_{\Phi}(0)=[0,1]$ is not internally chain transitive.

More generally one defines

$$
\omega_{\Phi}(Y):=\bigcap_{t \geq 0} \overline{\Phi_{[t, \infty)}(Y)}
$$

Definition A set $Y$ is forward precompact if $\overline{\Phi_{[t, \infty)}(Y)}$ is compact for some $t>0$.

Lemma 3.8 (i) $\omega_{\Phi}(Y)$ is the set of points $p \in \mathbb{R}^{m}$ such that

$$
p=\lim _{n \rightarrow \infty} \mathbf{y}_{n}\left(t_{n}\right)
$$

for some sequence $\left\{\mathbf{y}_{n}\right\}$ of solutions to $(I)$ with initial conditions $\mathbf{y}_{n}(0) \in$ $Y$ and some sequence $\left\{t_{n}\right\} \in \mathbb{R}$ with $t_{n} \rightarrow \infty$. 
(ii) $\omega_{\Phi}(Y)$ is a closed invariant (possibly empty) set. If $Y$ is forward precompact, then $\omega_{\Phi}(Y)$ is nonempty and compact.

Proof $(i)$ is easily seen from the definition.

(ii) Let $p=\lim _{n \rightarrow \infty} \mathbf{y}_{n}\left(t_{n}\right) \in \omega_{\Phi}(Y)$. Set $\mathbf{z}_{n}(s)=\mathbf{y}_{n}\left(t_{n}+s\right)$ for all $s \in \mathbb{R}$. By Lemma 3.1 we may extract from $\left(\mathbf{z}_{n}\right)_{n \geq 0}$ a subsequence converging to some solution $\mathbf{z}$ with $\mathbf{z}(0)=p$ and $\mathbf{z}(s)=\lim _{n_{k} \rightarrow \infty} \mathbf{y}_{n_{k}}\left(t_{n_{k}}+s\right) \in \omega_{\Phi}(Y)$. This proves invariance. The rest is clear. QED

Note that the limit set $\omega_{\Phi}(Y)$ is in general not strongly positively invariant (e.g., in Example 3.7 for $x<0, \omega_{\Phi}(x)=\{0\}$.)

\subsection{Attracting sets and attractors}

For applications it is useful to characterize $L(\mathbf{y})$ in terms of attractors for $\Phi$. Given a closed invariant set $L$, the induced set valued dynamical system $\Phi^{L}$ is the family of (set-valued) mappings $\Phi^{L}=\left\{\Phi_{t}^{L}\right\}_{t \in \mathbb{R}}$ defined on $L$ by

$$
\Phi_{t}^{L}(x)=\{\mathbf{x}(t): \mathbf{x} \text { is a solution to }(I) \text { with } \mathbf{x}(0)=x \text { and } \mathbf{x}(\mathbb{R}) \subset L\} .
$$

Note that $L$ is strongly invariant for $\Phi^{L}$.

Definition A compact set $A \subset L$ is called an attracting set for $\Phi^{L}$ provided there is a neighborhood $U$ of $A$ in $L$ (i.e for the induced topology) with the property that: for every $\varepsilon>0$ there exists $t_{\varepsilon}>0$ such that

$$
\Phi_{t}^{L}(U) \subset N^{\varepsilon}(A)
$$

for all $t \geq t_{\varepsilon}$. Or, equivalently, $\Phi_{\left[t_{\varepsilon}, \infty\right)}^{L}(U) \subset N^{\varepsilon}(A)$. Here $N^{\varepsilon}(A)$ stands for the $\varepsilon$-neighborhood of $A$.

If additionally $A$ is invariant, then $A$ is called an attractor.

The set $U$ is called a fundamental neighborhood of $A$ for $\Phi^{L}$. If $A \neq L$ and $A \neq \emptyset$ then $A$ is called a proper attracting set (or proper attractor) for $\Phi^{L}$.

Example 3.9 Let $F$ be the set-valued map from Example 3.2 (a), i.e., defined on $\mathbb{R}$ by $F(x)=-\operatorname{sgn}(x)$ if $x \neq 0$ and $F(0)=[-1,1]$. Then $\{0\}$ is an attractor and every compact set $A \subset \mathbb{R}$ with $0 \in A$ is an attracting set.

Proposition 3.10 Let $A$ be a non-empty compact subset of $L$ and $U$ a neighborhood of $A$ in L. Then 
(i) $A$ is an attracting set for $\Phi^{L}$ with fundamental neighborhood $U$ if and only if $U$ is forward precompact and $\omega_{\Phi^{L}}(U) \subset A$. In this case $\omega_{\Phi^{L}}(U)$ is an attractor.

(ii) $A$ is an attractor for $\Phi^{L}$ with fundamental neighborhood $U$ if and only if $U$ is forward precompact and $\omega_{\Phi^{L}}(U)=A$.

Proof $(i)$ If $A$ is an attracting set for $\Phi^{L}$ with fundamental neighborhood $U$ then $\omega_{\Phi^{L}}(U) \subset \bigcap_{\varepsilon>0} N^{\varepsilon}(A) \subset A$. Conversely, for $t$ large enough $V_{t}=\overline{\Phi_{[t, \infty)}^{L}(U)}$ defines a decreasing family of compact sets converging to $\omega_{\Phi^{L}}(U) \subset A$. Hence for any $\varepsilon>0$, there exists $t_{\varepsilon}$ with $V_{t_{\varepsilon}} \subset N^{\varepsilon}(A)$ and $A$ is an attracting set. In particular, $\omega_{\Phi^{L}}(U)$ itself is an attracting set, invariant by Lemma 3.8 (ii).

(ii) If $A=\omega_{\Phi^{L}}(U)$ then $A$ is an attractor by $(i)$. Conversely, if $A$ is an attractor with fundamental neighborhood $U$ then $\omega_{\Phi}(U) \subset A$ by $(i)$. Let $x \in A$. Since $A$ is invariant there exists a solution $\mathbf{y}$ to $(I)$ with $\mathbf{y}(0)=x$ and $\mathbf{y}(\mathbb{R}) \subset A$. Set $\mathbf{y}_{n}(t)=\mathbf{y}(t-n)$. Then $\mathbf{y}_{n}(n)=x$ proving that $x \in \omega_{\Phi^{L}}(U)$ (by Lemma 3.8, $(i))$. QED

Proposition 3.11 An attractor is strongly positively invariant.

(Example 3.2 (a) provides an attractor that is not strongly invariant.)

Proof By invariance $A \subset \Phi_{T}^{L}(A)$ for all $T>0$. Hence, given $t>0$

$$
\Phi_{t}^{L}(A) \subset \Phi_{t+T}^{L}(A) \subset \Phi_{t+T}^{L}(U) \subset \Phi_{[t+T, \infty)}^{L}(U)
$$

for all $T>0$. Thus $\Phi_{t}^{L}(A) \subset N^{\varepsilon}(A)$, for all $\varepsilon>0$; hence $\Phi_{t}^{L}(A) \subset A$ for all $t>0$. QED

Remark 3.12 In the family of attracting sets $A$ with a given fundamental neighborhood $U$, there exists a minimal one, which is in addition invariant, strongly positively invariant and independent of the set $U$ used to define the family. It is also the largest positively quasi-invariant set included in $U$.

Any attractor $A \subset L$ can be written as $A=\omega_{\Phi^{L}}(U)$ for some $U$. Hence any fundamental neighborhood uniquely determines the attractor $A$. This implies as in Conley (1978) that $\Phi^{L}$ can have at most countably many attractors. 


\subsection{Attractors and stability}

Definition. A set $A \subset L$ is asymptotically stable for $\Phi^{L}$ if it satisfies the following three conditions.

(i) $A$ is invariant.

(ii) $A$ is Lyapunov stable, i.e., for every neighborhood $U$ of $A$ there exists a neighborhood $V$ of $A$ such that $\Phi_{[0, \infty)}(V) \subset U$.

(iii) $A$ is attractive, i.e., there is a neighborhood $U$ of $A$ such that for every $x \in U: \omega_{\Phi}(x) \subset A$.

Alternatively, instead of (iii) one could ask for the weaker requirement

(iii') There is a neighborhood $U$ of $A$ such that for every solution $\mathbf{x}$ with $\mathbf{x}(0) \in U$ one has $L(\mathbf{x}) \subset A$.

We show now the equivalence of attractor and asymptotic stability. The proof of Corollary 3.18 below shows that it makes no difference whether one uses (iii) or (iii') in the definition of asymptotic stability.

We start with an upper bound for entry times.

Lemma 3.13 Let $V$ be an open set and $K$ compact such that for all solutions $\mathbf{x}$ with $\mathbf{x}(0) \in K$ there is $t>0$ with $\mathbf{x}(t) \in V$. Then there exists $T>0$ such that for every solution $\mathbf{x}$ with $\mathbf{x}(0) \in K$ there is $t \in[0, T]$ with $\mathbf{x}(t) \in V$.

Proof Suppose there is no such upper bound $T$ for the entry times into $V$. Then for each $n \in \mathbb{N}$ there is $\mathbf{x}_{n} \in K$ and a solution $\mathbf{x}_{n}$ such that $\mathbf{x}_{n}(t) \notin V$ for $0 \leq t \leq n$. Since $K$ is compact we can assume that $x_{n} \rightarrow x \in K$. And by Lemma 3.1 a subsequence of $\mathbf{x}_{n}$ converges to a solution $\mathbf{x}$ with $\mathbf{x}(0)=x$ and $\mathbf{x}(t) \notin V$ for all $t>0$. QED

Lemma 3.14 If a closed set $A$ is Lyapunov stable then it is strongly positively invariant.

Proof $A$ is the intersection of a family of strongly positively invariant neighborhoods. QED

Lemma 3.15 If a compact set A satisfies (ii) and (iii'), it is attracting. 
Proof Let $B$ be a compact neighborhood of $A$, included in the fundamental neighborhood $U$ and let $W$ be a neighborhood of $A$. $A$ being Lyapunov stable, there exists an open neighborhood $V$ of $A$ with $\Phi_{[0, \infty)}^{L}(V) \subset W$. For any $x \in B$ and any solution $\mathbf{x}$ with $\mathbf{x}(0)=x$, there exists $t>0$ with $\mathbf{x}(t) \in V$. Applying Lemma 3.13 implies $\Phi_{T}^{L}(B) \subset \Phi_{[0, T]}^{L}(V)$ hence $\Phi_{[T, \infty)}^{L}(B) \subset W$ and $A$ is attracting. QED

Lemma 3.16 If a compact set $A$ is attracting and strongly positively invariant it is Lyapunov stable.

Proof Let $A$ be attracting with fundamental neighborhood $U$, and $V$ be any other (open) neighborhood of $A$. Then by definition there is $T>0$ such that $\Phi_{[T, \infty)}^{L}(U) \subset V . A$ being strongly positively invariant $\phi_{[0, T]}^{L}(A) \subset A$. Uppersemicontinuity gives an $\varepsilon>0$ such that $\phi_{[0, T]}^{L}\left(N^{\varepsilon}(A)\right) \subset V$ and $N^{\varepsilon}(A) \subset U$. Hence $\Phi_{[0, \infty)}^{L}\left(N^{\varepsilon}(A)\right) \subset V$ which shows Lyapunov stability. QED

Corollary 3.17 Properties (ii) and (iii') are equivalent to attracting and strong positive invariance.

Corollary 3.18 $A$ compact set $A$ is an attractor if and only if it is asymptotically stable.

We conclude with a simple useful condition ensuring that an open set contains an attractor.

Proposition 3.19 Let $U$ be an open set with compact closure. Suppose that $\Phi_{T}(\bar{U}) \subset U$ for some $T>0$. Then $U$ is a fundamental neighborhood of an attractor $A$.

Proof Since $\Phi$ has a closed graph $\Phi_{T}(\bar{U})$ is compact. Therefore $\Phi_{T}(\bar{U}) \subset V \subset$ $\bar{V} \subset U$ for some open set $V$. By upper semi continuity of $\Phi_{T}$ (which follows from property (d) of a set-valued dynamical system) there exists $\varepsilon>0$ such that $\Phi_{t}(\bar{U}) \subset V$ for $T-\varepsilon \leq t \leq T+\varepsilon$. Let $t_{0}=T(T+1) / \varepsilon$. For all $t \geq t_{0}$ write $t=k T+r$ with $k \in \mathbb{N}$ and $r<T$. Hence $t=k(T+r / k)$ with $0 \leq r / k<\varepsilon$. Thus

$$
\Phi_{t}(\bar{U})=\Phi_{T+r / k} \circ \ldots \circ \Phi_{T+r / k}(\bar{U}) \subset V .
$$

Hence $\omega_{\Phi}(U)=\bigcap_{t>t_{0}} \overline{\Phi_{[t, \infty)}(U)} \subset \bar{V} \subset U$ is an attractor with fundamental neighborhood $U$. QED 


\subsection{Chain transitivity and attractors}

Proposition 3.20 Let $L$ be internally chain-transitive. Then $\Phi^{L}$ has no proper attracting set.

Proof Let $A \subset L$ be an attracting set and $U, t_{\varepsilon}$ as in the definition. Assume $A \neq L$ and choose $\varepsilon$ small enough so that $N^{2 \varepsilon}(A) \subset U$ and there exists $y \in L \backslash N^{2 \varepsilon}(A)$. Then, for $T \geq t_{\varepsilon}$ and $x \in A$, there is no $(\varepsilon, T)$ chain from $x$ to $y$. In fact, $\mathbf{x}_{1}(0) \in N^{2 \varepsilon}(A)$, hence $\mathbf{x}_{1}\left(t_{1}\right) \in N^{\varepsilon}(A)$; by induction $\mathbf{x}_{i}\left(t_{i}\right) \in N^{\varepsilon}(A)$ so that $\mathbf{x}_{i+1}(0) \in N^{2 \varepsilon}(A)$ as well. A contradiction. QED

Remark 3.21 This later proposition can also be deduced from Theorem 1 in Bronstein and Kopanskii (1988) combined with Lemma 3.1. Also the converse is true.

An attracting set (respectively attractor) for $\Phi$ is an attracting set (respectively attractor) for $\Phi^{L}$ with $L=\mathbb{R}^{m}$.

Lemma 3.22 Let $A$ be an attracting set for $\Phi$ and $L$ a closed invariant set. Assume $A \cap L \neq \emptyset$. Then $A \cap L$ is an attracting set for $\Phi^{L}$.

Proof follows from the definitions. QED

If $A$ is a set then

$$
B(A)=\left\{x \in \mathbb{R}^{m}: \omega_{\Phi}(x) \subset A\right\}
$$

denote its basin of attraction.

Theorem 3.23 Let $A$ be an attracting set for $\Phi$ and $L$ an internally chain transitive set. Assume $L \cap B(A) \neq \emptyset$. Then $L \subset A$.

Proof Suppose $L \cap B(A) \neq \emptyset$. Then there exists a solution $\mathbf{x}$ to $(I)$ with $\mathbf{x}(0)=x \in B(A)$ and $\mathbf{x}(\mathbb{R}) \subset L$. Hence $d(\mathbf{x}(t), A) \rightarrow 0$ when $t \rightarrow \infty$ proving that $L$ meets $A$. Proposition 3.20 and Lemma 3.22 imply that $L \subset A$. QED

A global attractor for $\Phi$ is an attractor whose basin of attraction consists of all $\mathbb{R}^{m}$. If a global attractor exists then it is unique and coincides with the maximal compact invariant set of $\Phi$. The following corollary is an immediate consequence of Theorem 3.23 or even easier of Lemma 3.5.

Corollary 3.24 Suppose $\Phi$ has a global attractor A. Then every internally chain transitive set lies in $A$. 


\subsection{Lyapunov Functions}

Proposition 3.25 Let $\Lambda$ be a compact set, $U \subset \mathbb{R}^{m}$ be a bounded open neighborhood of $\Lambda$ and $V: \bar{U} \rightarrow[0, \infty[$. Let

(i) For all $t \geq 0, \Phi_{t}(U) \subset U$, (i.e., $U$ is strongly positively invariant)

(ii) $V^{-1}(0)=\Lambda$,

(iii) $V$ is continuous and for all $x \in U \backslash \Lambda, y \in \Phi_{t}(x)$ and $t>0, V(y)<V(x)$.

(iv) $V$ is upper semi continuous and for all $x \in \bar{U} \backslash \Lambda, y \in \Phi_{t}(x)$ and $t>0$, $V(y)<V(x)$.

a) Under $(i),($ ii $)$ and (iii), $\Lambda$ is a Lyapunov stable attracting set and there exists an attractor contained in $\Lambda$ whose basin contains $U$, and with $V^{-1}([0, r))$ as fundamental neighborhoods for small $r>0$.

b) Under $(i),(i i)$ and $(i v)$, there exists an attractor contained in $\Lambda$ whose basin contains $U$.

Proof For the proof of $a$ ), let $r>0$ and $U_{r}=\{x \in U: V(x)<r\}$. Then $\left\{\bar{U}_{r}\right\}_{r>0}$ is a nested family of compact neighborhoods of $\Lambda$ with $\bigcap_{r>0} \bar{U}_{r}=\Lambda$. Thus for $r>0$ small enough, $\overline{U_{r}} \subset U$. Moreover $\Phi_{t}\left(\bar{U}_{r}\right) \subset U_{r}$ for $t>0$ by our hypotheses on $U$ and $V$. Proposition 3.19 then implies the result.

For $b$ ), let $A=\omega_{\Phi}(U)$ which is closed and invariant (Lemma 3.7), hence compact, since included in $\bar{U}$. Let $\alpha=\max _{y \in A} V(y)$ reached at $x$, since $V$ is scs. By invariance there exists $\mathbf{x}$ solution and $t>0$ with $z=\mathbf{x}(0) \in A$ and $\mathbf{x}(t)=x$. This contradicts $(i v)$ unless $\alpha=0$ and $A \subset \Lambda$. Thus $U$ is a neighborhood of $A$ which is an attractor included in $\Lambda$. QED

Remark 3.26 One can show that to every attractor $A$ there exists a function $V$ such that Proposition $3.25(i v)$ holds for $\Lambda=A$. Take $V(x)=\max \{d(y, A) g(t) ; y \in$ $\left.\Phi_{t}(x), t \geq 0\right\}$. where $d>g(t)>c>0$ is any continuous strictly increasing function.

Let $\Lambda$ be any subset of $\mathbb{R}^{m}$. A continuous function $V: \mathbb{R}^{m} \rightarrow \mathbb{R}$ is called a Lyapunov function for $\Lambda$ if $V(y)<V(x)$ for all $x \in \mathbb{R}^{m} \backslash \Lambda, y \in \Phi_{t}(x), t>0$; and $V(y) \leq V(x)$ for all $x \in \Lambda, y \in \Phi_{t}(x)$ and $t \geq 0$. Note that for each solution $\mathbf{x}, V$ is constant along its limit set $L(\mathbf{x})$.

The following result is similar to Proposition 6.4 in Benaïm (1999). 
Proposition 3.27 Suppose $V$ is a Lyapunov function for $\Lambda$. Assume that $V(\Lambda)$ has empty interior. Then every internally chain transitive set $L$ is contained in $\Lambda$ and $V \mid L$ is constant.

Proof Let

$$
v=\inf \{V(y): y \in L\} .
$$

Since $L$ is compact and $V$ is continuous, $v=V(x)$ for some point $x \in L$. Since $L$ is invariant there exists a solution $\mathbf{x}$ with $\mathbf{x}(t) \in L$ and $\mathbf{x}(0)=x$. Then $v=V(x)>V(\mathbf{x}(t))$ is impossible for $t>0$. Since $\mathbf{x}(t) \in \Phi_{t}(x)$, we conclude $x \in \Lambda$.

Thus $v$ belongs to the range $V(\Lambda)$. Since $V(\Lambda)$ contains no interval there is a sequence $v_{n} \notin V(\Lambda)$ decreasing to $v$. The sets $L_{n}=\left\{x \in L: V(x)<v_{n}\right\}$ satisfy $\Phi_{t}\left(\bar{L}_{n}\right) \subset L_{n}$ for $t>0$. In fact either $x \in \Lambda \cap \bar{L}_{n}$ and $V(y) \leq V(x)<v_{n}$ or $V(y)<V(x) \leq v_{n}$, for any $y \in \Phi_{t}(x), t>0$.

Thus using Proposition 3.19 and Proposition 3.20 one obtains $L=\bigcap_{n} \bar{L}_{n}=$ $\{x \in L: V(x)=v\}$. Thus $V$ is constant on $L$. $L$ being invariant this implies as above $L \subset \Lambda$. QED

Corollary 3.28 Let $V$ and $\Lambda$ be as in Proposition 3.27. Suppose furthermore that $V$ is $C^{m}$ and $\Lambda$ is contained in the critical points set of $V$. Then every internally chain transitive set lies in $\Lambda$ and $V \mid L$ is constant.

Proof By Sard's theorem (Hirsch, 1976, p. 69) $V(\Lambda)$ has empty interior and Proposition 3.27 applies. QED

\section{The limit set Theorem}

\subsection{Asymptotic pseudo trajectories for set-valued dy- namics}

The translation flow $\Theta: C^{0}\left(\mathbb{R}, \mathbb{R}^{m}\right) \times \mathbb{R} \rightarrow C^{0}\left(\mathbb{R}, \mathbb{R}^{m}\right)$ is the flow defined by

$$
\Theta^{t}(\mathbf{x})(s)=\mathbf{x}(s+t)
$$

A continuous function $\mathbf{z}: \mathbb{R}^{+} \rightarrow \mathbb{R}^{m}$ is an asymptotic pseudo trajectory (APT) for $\Phi$ if

$$
\lim _{t \rightarrow \infty} \mathbf{D}\left(\Theta^{t}(\mathbf{z}), S_{\mathbf{z}(t)}\right)=0
$$


(or $\lim _{t \rightarrow \infty} \mathbf{D}\left(\Theta^{t}(\mathbf{z}), S\right)=0$, where $S=\bigcup_{x \in \mathbb{R}^{m}} S_{x}$ denotes the set of all solutions of (I).)

Alternatively, for all $T$

$$
\lim _{t \rightarrow \infty} \inf _{\mathbf{x} \in S_{\mathbf{z}(t)}} \sup _{0 \leq s \leq T}\|\mathbf{z}(t+s)-\mathbf{x}(s)\|=0
$$

In other words, for each fixed $T$, the curve

$$
[0, T] \rightarrow \mathbb{R}^{m}: s \rightarrow \mathbf{z}(t+s)
$$

shadows some $\Phi$ trajectory of the point $\mathbf{z}(t)$ over the interval $[0, T]$ with arbitrary accuracy for sufficient large $t$. Hence $\mathbf{z}$ has a forward trajectory under $\Theta$ attracted by $S$. As usual one extends $\mathbf{z}$ to $\mathbb{R}$ by letting $\mathbf{z}(t)=\mathbf{z}(0)$ for $t<0$.

The next result is a natural extension of Theorem 7.2 in Benaïm and Hirsch (1996).

Theorem 4.1 (Characterization of APT)

Assume $\mathbf{z}$ is bounded. Then there is equivalence between

(i) $\mathbf{z}$ is an $A P T$ for $\Phi$.

(ii) $\mathbf{z}$ is uniformly continuous and any limit point of $\left\{\Theta^{t}(\mathbf{z})\right\}$ is in $S$.

In both cases the set $\left\{\Theta^{t}(\mathbf{z}) ; t \geq 0\right\}$ is relatively compact.

Proof By hypothesis, $K=\overline{\{\mathbf{z}(t) ; t \geq 0\}}$ is compact.

For any $\varepsilon>0$, there exists $\eta>0$ such that $\|z-x\|<\varepsilon / 2$, for any $x \in K$, any $z \in \Phi_{s}(x)$, and any $|s|<\eta$ using property (d) of the dynamical system.

$\mathbf{z}$ being an APT, there exists $T$ such that $t>T$ implies

$$
d\left(\mathbf{z}(t+s), \Phi_{s}(\mathbf{z}(t))\right)<\varepsilon / 2, \quad \forall|s|<\eta
$$

hence

$$
\|\mathbf{z}(t+s)-\mathbf{z}(t)\| \leq \varepsilon
$$

and $\mathbf{z}$ is uniformly continuous. Clearly any limit point belongs to $S$ by the condition (4) above.

Conversely, if $\mathbf{z}$ is uniformly continuous, then the family of functions $\left\{\Theta^{t}(\mathbf{z}) ; t \geq\right.$ $0\}$ is equicontinuous and hence ( $K$ being compact) relatively compact by Ascoli's theorem. Since any limit point belongs to $S$, property (4) follows. QED 


\subsection{Perturbed solutions are APT}

Theorem 4.2 Any bounded solution $\mathbf{y}$ of $(I I)$ is an APT of $(I)$.

Proof Let us prove that $\mathbf{y}$ satisfies condition (ii) in the previous Theorem. Set $v(t)=\dot{\mathbf{y}}(t)-U(t) \in F^{\delta(t)}(\mathbf{y}(t))$. Then,

$$
\mathbf{y}(t+s)-\mathbf{y}(t)=\int_{0}^{s} v(t+\tau) d \tau+\int_{t}^{t+s} U(\tau) d \tau
$$

By assumption (iii) of $(I I)$, the second integral goes to 0 , as $t \rightarrow \infty$. The boundedness of $\mathbf{y}, \mathbf{y}(\mathbb{R}) \subset M, M$ compact (combined with the fact that $F$ has linear growth) implies boundedness of $v$ and shows that $\mathbf{y}$ is uniformly continuous. Thus the family $\Theta^{t}(\mathbf{y})$ is equicontinuous, hence relatively compact. Let $\mathbf{z}=\lim _{t_{n} \rightarrow \infty} \Theta^{t_{n}}(\mathbf{y})$ be a limit point. Set $t=t_{n}$ in (5) and define $v_{n}(s)=v\left(t_{n}+s\right)$. Then, using the assumption (iii) on $U$, the second term in the right hand side of this equality goes to zero uniformly on compact intervals when $n \rightarrow \infty$. Hence

$$
\mathbf{z}(s)-\mathbf{z}(0)=\lim _{n \rightarrow \infty} \int_{0}^{s} v_{n}(\tau) d \tau
$$

Since $\left(v_{n}\right)$ is uniformly bounded, it is bounded in $L^{2}[0, s]$ and by the BanachAlaoglu theorem, a subsequence of $v_{n}$ will converge weakly in $L^{2}[0, s]$ (or weak* in $\left.L^{\infty}[0, s]\right)$ to some function $v$ with $v(t) \in F(\mathbf{z}(t))$, for almost every $t$, since $v_{n}(t) \in F^{\delta\left(t+t_{n}\right)}\left(\mathbf{y}\left(t+t_{n}\right)\right)$ for every $t$. Here we use (ii) and that $F$ is upper semi continuous with convex values. In fact by Mazur's theorem a convex combination of $\left\{v_{m}, m \geq n\right\}$ converge a.s. to $v$ and $\lim _{m \rightarrow \infty} C o\left(\bigcup_{n \geq m} F^{\delta\left(t+t_{n}\right)}(\mathbf{y}(t+\right.$ $\left.\left.\left.t_{n}\right)\right)\right) \subset F(\mathbf{z}(t))$. Hence $\mathbf{z}(s)-\mathbf{z}(0)=\int_{0}^{s} v(\tau) d \tau$, proving that $\mathbf{z}$ is a solution of $(I)$ and hence $\mathbf{z} \in S_{M, M}$. QED

\subsection{APT are ICT}

Theorem 4.3 Let $\mathbf{z}$ be a bounded APT of $(I)$. Then $L(\mathbf{z})$ is internally chain transitive.

Proof The set $\left\{\Theta^{t}(\mathbf{z}): t \geq 0\right\}$, is relatively compact, hence the $\omega$-limit set of $\mathbf{z}$ for the flow $\Theta$

$$
\omega_{\Theta}(\mathbf{z})=\bigcap_{t \geq 0} \overline{\left\{\Theta^{s}(\mathbf{z}): s \geq t\right\}} .
$$


is internally chain transitive (by standard properties of $\omega$-limit sets of bounded semiorbits, $\omega_{\Theta}(\mathbf{z})$ is a nonempty, compact, internally chain transitive set invariant under $\Theta$, see Conley (1978) ; a short proof is in Benaïm (1999), Corollary 5.6). By property (4), $\omega_{\Theta}(\mathbf{z}) \subset S$, the set of all solutions of $(I)$.

Let $\Pi:\left(C^{0}\left(\mathbb{R}, \mathbb{R}^{m}\right), \mathbf{D}\right) \rightarrow\left(\mathbb{R}^{m},\|\cdot\|\right)$ be the projection map defined by $\Pi(\mathbf{z})=$ $\mathbf{z}(0)$. One has $\Pi\left(\omega_{\Theta}(\mathbf{z})\right)=L(\mathbf{z})$. In fact if $p=\lim _{n \rightarrow \infty} \mathbf{z}\left(t_{n}\right)$, let $\mathbf{w}$ be a limit point of $\Theta^{t_{n}}(\mathbf{z})$. Then $\mathbf{w} \in \omega_{\Theta}(\mathbf{z})$ and $\Pi(\mathbf{w})=p$.

It then easily follows that $L(\mathbf{z})$ is nonempty compact and invariant under $\Phi$ since $\omega_{\Theta}(\mathbf{z}) \subset S$. Since $\Pi$ has Lipschitz constant $1, \Pi$ maps every $(\varepsilon, T)$ chain for $\Theta$ to an $(\varepsilon, T)$ chain for $\Phi$. This proves that $L(\mathbf{z})$ is internally chain transitive for $\Phi$. QED

\section{Applications}

\subsection{Approachability}

An application of Proposition 3.25 is the following result which can be seen as a continuous asymptotic deterministic version of Blackwell's approachability theorem (Blackwell, 1956). Note that one has no property on uniform speed of convergence.

Given a compact set $\Lambda \in \mathbb{R}^{m}$ and $x \in \mathbb{R}^{m}$ we let $\Pi_{\Lambda}(x)=\left\{y \in \Lambda: d^{2}(x, \Lambda)=\right.$ $\left.\|x-y\|^{2}=\langle x-y, x-y\rangle\right\}$.

Corollary 5.1 Let $\Lambda \subset \mathbb{R}^{m}$ be a compact set, $r>0$ and $U=\left\{x \in \mathbb{R}^{m}\right.$ : $d(x, \Lambda)<r\}$. Suppose that for all $x \in U \backslash \Lambda$ there exists $y \in \Pi_{\Lambda}(x)$ such that the affine hyperplane orthogonal to $[x, y]$ at $y$ separates $x$ from $x+F(x)$. That is

$$
\langle x-y, x-y+v\rangle \leq 0
$$

for all $v \in F(x)$. Then $\Lambda$ contains an attractor for (I) with fundamental neighborhood $U$.

Proof Set $V(x)=d(x, \Lambda)$. To apply Proposition 3.25 it suffices to verify condition (iii) of Proposition 3.25. Condition ( $i$ ) will follow and condition ( $i i)$ is clearly true.

Let $\mathbf{x}$ be a solution to $(I)$ with initial condition $x \in U \backslash \Lambda$. Set $\tau=\inf \{t>$ $0: \mathbf{x}(t) \in \Lambda\} \leq \infty, g(t)=V(\mathbf{x}(t))$ and let $I \subset[0, \tau[$ be the set of $0 \leq t<\tau$ such that $g^{\prime}(t)$ and $\dot{\mathbf{x}}(t)$ exist and $\dot{\mathbf{x}}(t) \in F(\mathbf{x}(t))$. For all $t \in I$ and $y \in \Pi_{\Lambda}(\mathbf{x}(t))$

$$
\begin{aligned}
& g(t+h)-g(t) \leq\|\mathbf{x}(t+h)-y\|-\|\mathbf{x}(t)-y\| \\
& =\|\mathbf{x}(t)+\dot{\mathbf{x}}(t) h-y\|-\|\mathbf{x}(t)-y\|+|h| \varepsilon(h)
\end{aligned}
$$


where $\lim _{h \rightarrow 0} \varepsilon(h)=0$. Hence

$$
\begin{gathered}
g^{\prime}(t) \leq \frac{1}{\|\mathbf{x}(t)-y\|}\langle\mathbf{x}(t)-y, \dot{\mathbf{x}}(t)\rangle \\
=-g(t)+\frac{1}{\|\mathbf{x}(t)-y\|}\langle\mathbf{x}(t)-y, \mathbf{x}(t)-y+\dot{\mathbf{x}}(t)\rangle .
\end{gathered}
$$

Thus, $\dot{x} \in F(x)$ and (6) imply $g^{\prime}(t) \leq-g(t)$ for all $t \in I$. Since $g$ and $\mathbf{x}$ are

absolutely continuous, $I$ has full measure in $\left[0, \tau\left[\right.\right.$. Hence $g(t) \leq e^{-t} g(0)$ for all $t<\tau$. Therefore $V(\mathbf{x}(t))<V(x)$ for all $0<t<\tau$, which shows (iii). Finally $V(\mathbf{x}(t)) \leq e^{-t} V(x)$ shows that the sets $V^{-1}\left[0, r^{\prime}\right)\left(\right.$ with $\left.0<r^{\prime} \leq r\right)$ are fundamental neighborhoods of the attractor in $\Lambda$. QED

In particular, if any point of $E$ has a unique projection on $\Lambda$ (for example $\Lambda$ convex) $\bar{C}=C$ and one recovers exactly Blackwell's sufficient condition for approachability.

Corollary 5.2 (Blackwell's approachability theorem). Consider the decision making process described in section 2.1, example 2.2. Let $\Lambda \subset E$ be a compact set. Assume that there exists a strategy $Q$ such that for all $x \in E \backslash \Lambda$ there exists $y \in \Pi_{\Lambda}(x)$ such that the hyperplane orthogonal to $[x, y]$ through $y$ separates $x$ from $\bar{C}(x)$. Then $\Lambda$ is approachable.

Proof Let $L\left(x_{n}\right)$ denote the limit set of $\left\{x_{n}\right\}$. Theorem 3.6 with Proposition 2.1, Corollary 5.1 and Corollary 3.24 imply that $L\left(x_{n}\right)$ is almost surely contained in $\Lambda$. QED

\subsection{Smale's approach to prisoner's dilemma}

We develop here example 2.4. Consider a $2 \times 2$ "Prisoner's Dilemma game". Each player has two possible actions: Cooperate (play C) or Defect (play D). If both cooperate, each receives $\alpha$; If both defect each receives $\lambda$; if one cooperates and the other defects the cooperator receives $\beta$ and the defector $\gamma$. We suppose that $\gamma>\alpha>\lambda>\beta$ as it is usual with a prisoner's dilemma game. We furthermore assume that

$$
\gamma-\alpha<\alpha-\beta
$$

so that the outcome space $E$ is the convex quadrilateral whose vertices are the payoff vectors

$$
\mathbf{C D}=(\beta, \gamma), \mathbf{C C}=(\alpha, \alpha), \mathbf{D C}=(\gamma, \beta), \mathbf{D D}=(\lambda, \lambda)
$$




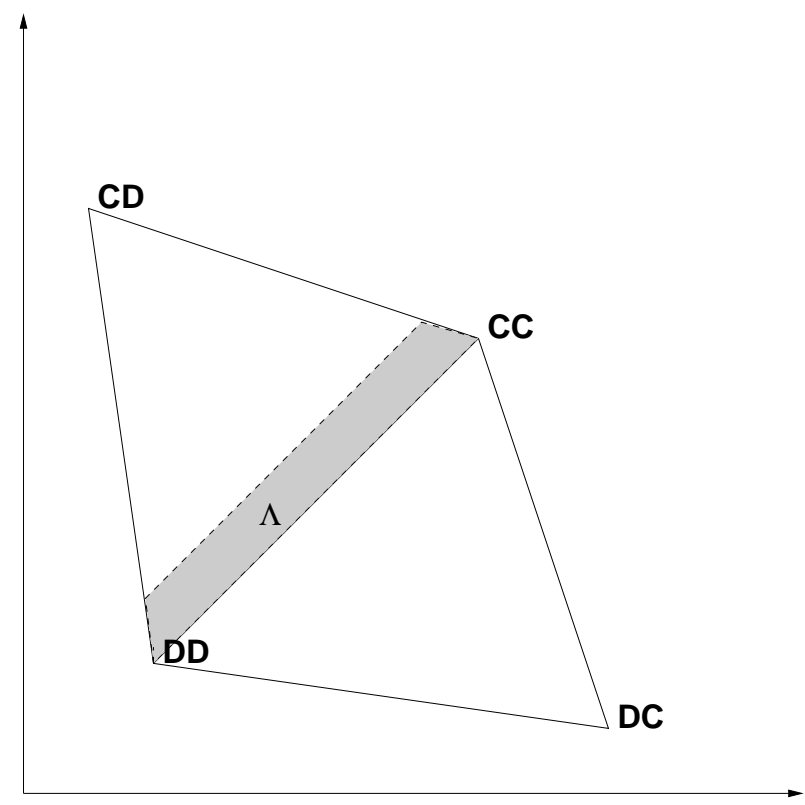

The outcome space $E$

Let $\delta$ be a nonnegative parameter. Adapting Smale (1980) and Benaïm and Hirsch (1996), a $\delta$-good strategy for player 1 is a strategy $Q^{1}=\left\{Q_{x}^{1}\right\}$ (as defined in section 2.1) enjoying the following features:

$$
Q_{x}^{1}(\text { play } \mathrm{C})=1 \text { if } x^{1}>x^{2},
$$

and

$$
Q_{x}^{1}\left(\text { play C) }=0 \text { if } x^{1}<x^{2}-\delta .\right.
$$

The following result reinterprets the results of Smale (1980) and Benaïm and Hirsch (1996) in the framework of approachability. It also provides some generalization (see the remark 5.4 below).

Theorem 5.3 (i) Suppose that player 1 plays a $\delta$-good strategy. Then the set

$$
\Lambda=\left\{x \in E: x^{2}-\delta \leq x^{1} \leq x^{2}\right\}
$$

is approachable.

(ii) Suppose that both players play a $\delta$-good strategy and that at least one of them is continuous (meaning that the corresponding function $x \rightarrow$ $Q_{x}^{i}($ play $C)$ is continuous). Then

$$
\lim _{n \rightarrow \infty} x_{n}=\mathbf{C C}
$$

almost surely. 
Proof $(i)$. Let $x \in E \backslash \Lambda$. If $x^{1}>x^{2}$ then

$$
C(x)=\bar{C}(x)=[\mathbf{C C}, \mathbf{C D}]
$$

and the line $\left\{u \in \mathbb{R}^{2}: u^{1}=u^{2}\right\}$ separates $x$ from $\bar{C}(x)$. Similarly if $x^{1}<x^{2}-\delta$ then

$$
C(x)=\bar{C}(x)=[\mathbf{D D}, \mathbf{D C}]
$$

which is separated from $x$ by the line $\left\{u \in \mathbb{R}^{2}: u^{1}=u^{2}-\delta\right\}$. Assertion $(i)$ then follows from Corollary 5.2.

(ii). If both play a $\delta$-good strategy, then $(i)$ and its analogue for player 2 implies that the diagonal

$$
\Delta=\left\{x \in E: x^{1}=x^{2}\right\}
$$

is approachable. Thus $L\left(x_{n}\right) \subset \Delta$. Also (by Proposition 2.1, Theorem 3.6 and Lemma 3.5) $L\left(x_{n}\right)$ is invariant under the differential inclusion induced by

$$
F(x)=-x+\bar{C}(x)
$$

where $C(x)=C^{1}(x) \cap C^{2}(x)$ and $C^{i}(x)$ is the convex set associated to $Q^{i}$ (the strategy of player $i$ ). Suppose that one player, say 1, plays a continuous strategy. Then $\bar{C}(x) \subset \overline{C^{1}}(x)=C^{1}(x)$ and for all $x \in \Delta, C^{1}(x)=[\mathbf{C D}, \mathbf{C C}]$. Now, there is only one subset of $\Delta$ which is invariant under $\dot{x} \in-x+[\mathbf{C D}, \mathbf{C C}]$; this is the point CC. This proves that $L\left(x_{n}\right)=$ CC. QED

Remark 5.4 (i) In contrast to Smale (1980) and Benaïm and Hirsch (1996) observe that assertion $(i)$ makes no hypothesis on player 2's behavior. In particular it is unnecessary to assume that player 2 has a strategy of the form defined by section 2.1 .

(ii) The regularity assumptions (on strategies) are much weaker than in Benaïm and Hirsch (1996).

(iii) A 0 -good strategy makes the diagonal $\Delta$ approachable. However if both players play a 0 -good strategy, then $\bar{C}(x)=E$ for all $x \in \Delta$ and we are unable to predict the long term behavior of $\left\{x_{n}\right\}$ on $\Delta$.

\subsection{Fictitious Play in Potential Games}

Here we generalize the result of Monderer and Shapley (1996). They prove convergence of the classical discrete fictitious play process, as defined in Example 2.3, for $n$-linear payoff functions. Harris (1998) studies the best response 
dynamics in this case, but does not derive convergence of fictitious play from it. Our limit set theorem provides the right tool for doing this, even in the following, more general setting.

Let $X_{i}, i=1, \ldots n$ be compact convex subsets of Euclidean spaces and $U: X_{1} \times \cdots \times X_{n} \rightarrow \mathbb{R}$ be a $C^{1}$ function which is concave in each variable. $U$ is interpreted as the common payoff function for the $n$ players. We write $x=\left(x_{i}, x_{-i}\right)$ and define $B R_{i}\left(x_{-i}\right):=\operatorname{Argmax}_{x_{i} \in X_{i}} U(x)$ the set of maximizers. Then $x \mapsto B R(x)=\left(B R_{1}\left(x_{-1}\right), \ldots B R_{n}\left(x_{-n}\right)\right)$ is u.s.c. (by Berge's (1966) Maximum Theorem, since $U$ is continuous) with nonempty compact convex values. Consider the best response dynamics

$$
\dot{\mathbf{x}} \in B R(\mathbf{x})-\mathbf{x} .
$$

Its constant solutions $\mathbf{x}(t) \equiv \hat{x}$ are precisely the Nash equilibria $\hat{x} \in B R(\hat{x})$, i.e., $U(\hat{x}) \geq U\left(x_{i}, \hat{x}_{-i}\right)$ for all $i$ and $x_{i} \in X_{i}$. Along a solution $\mathbf{x}(t)$ of $(7)$, let $u(t)=U(\mathbf{x}(t))$. Then for almost all $t>0$,

$$
\begin{aligned}
\dot{u}(t) & =\sum_{i=1}^{n} \frac{\partial U}{\partial x_{i}}(\mathbf{x}(\mathbf{t})) \dot{\mathbf{x}}_{i}(t) \\
& \geq \sum_{i=1}^{n}\left[U\left(\mathbf{x}_{i}(t)+\dot{\mathbf{x}}_{i}(t), \mathbf{x}_{-i}(t)\right)-U(\mathbf{x}(t))\right] \\
& =\sum_{i=1}^{n}\left[\max _{y_{i} \in X_{i}} U\left(y_{i}, \mathbf{x}_{-i}(t)\right)-U(\mathbf{x}(t))\right] \geq 0 .
\end{aligned}
$$

where from (8) to (9) we use the concavity of $U$ in $x_{i}$, and (10) follows from (7) and the definition of $B R_{i}$. Since the function $t \mapsto u(t)$ is locally Lipschitz, this shows that it is weakly increasing. It is constant in a time interval $T$, if and only if $\mathbf{x}_{i}(t) \in B R_{i}\left(\mathbf{x}_{-i}(t)\right)$ for all $t \in T$ and $i=1, \ldots n$, i.e., iff $\mathbf{x}(t)$ is a Nash equilibrium for $t \in T$ (but $\mathbf{x}(t)$ may move in a component of NE with constant $U)$.

Theorem 5.5 The limit set of every solution of (7) is a connected set of NE, along which $U$ is constant. If furthermore the set $U(N E)$ contains no interval in $\mathbb{R}$ then the limit set of every fictitious play path is a connected set of $N E$ along which $U$ is constant.

Proof The first statement follows from above. The second statement follows from Theorem 3.6 together with Propositon 3.27 with $V=-U$ and $\Lambda$ the set of NE. 
Remark 5.6 The assumption that the set $U(N E)$ contains no interval in $\mathbb{R}$ follows via Corollary 3.28 if $U$ is smooth enough (e.g., in the $n$-linear case) and if each $X_{i}$ has at most countably many faces, by applying Sard's lemma in the interior of each face.

Example 5.7 $2 \times 2$ coordination game. The global attractor of (7) consists of three equilibria and two line segments connecting them. The internally chain transitive sets are the three equilibria. Hence every fictitious play process converges to one of these equilibria.

The case of (continuous concave-convex) two-person zero-sum games was treated in Hofbauer and Sorin (2002) where it is shown that the global attractor of (7) equals the set of equilibria. In this case the full strength of Theorem 3.6 and the notion of chain transitivity are not needed, the invariance of the limit set of a fictitious play path implies that it is contained in the global attractor, compare Corollary 3.24.

\section{References}

[1] J.-P. Aubin and A. Cellina, (1984) Differential Inclusions, Springer.

[2] M. Benaïm, (1996) A dynamical system approach to stochastic approximation, SIAM Journal on Control and Optimization, 34, 437-472.

[3] M. Benaïm, (1999) Dynamics of stochastic approximation algorithms, Séminaire de Probabilités XXXIII, Lecture Notes in Math. 1709, 1-68, Springer.

[4] M. Benaïm and M.W. Hirsch, (1996a) Stochastic adaptive behavior for prisoner's dilemma, preprint.

[5] M. Benaïm and M.W. Hirsch, (1996b) Asymptotic pseudotrajectories and chain recurrent flows, with applications, J. Dynam. Differential Equations, 8, 141-176.

[6] M. Benaïm and M.W. Hirsch, (1999) Mixed equilibria and dynamical systems arising from fictitious play in perturbed games, Games and Economic Behavior, 29, 36-72.

[7] M. Benaïm, J. Hofbauer and S. Sorin (2003) Stochastic approximations and differential inclusions: applications, in preparation. 
[8] D. Blackwell, (1956) An analog of the minmax theorem for vector payoffs, Pacific Journal of Mathematics, 6, 1-8.

[9] I. U. Bronstein and A. Ya. Kopanskii, (1988) Chain recurrence in dynamical systems without uniqueness, Nonlinear Analysis, Theory and Applications, 12, 147-154 .

[10] G. Brown, (1951) Iterative solution of games by fictitious play, in Koopmans T.C. (ed.) Activity Analysis of Production and Allocation, Wiley, 374-376.

[11] F.H. Clarke, Yu. S. Ledyaev, R.J. Stern and P.R. Wolenski, (1998) Nonsmooth Analysis and Control Theory, Springer.

[12] C. C. Conley, (1978) Isolated invariant sets and the Morse index, CBMS Regional conference series in mathematics, 38, American Mathematical Society, Providence.

[13] M. Duflo, (1996) Algorithmes Stochastiques, Springer.

[14] D. Fudenberg and D. K. Levine, (1998) The Theory of Learning in Games, MIT Press.

[15] I. Gilboa and A. Matsui, (1991) Social stability and equilibrium, Econometrica, 59, 859-867.

[16] M.W. Hirsch, (1976) Differential Topology, Springer.

[17] J. Hofbauer, (1995) Stability for the best response dynamics, mimeo.

[18] J. Hofbauer and W.H. Sandholm, (2002) On the global convergence of stochastic fictitious play, Econometrica, 70 , 2265-94.

[19] J. Hofbauer and S. Sorin, (2002) Best response dynamics for continuous zero-sum games, Cahier du Laboratoire d'Econometrie, Ecole Polytechnique, 2002-028.

[20] H.J. Kushner and G.G. Yin, (1997) Stochastic Approximations Algorithms and Applications, Springer.

[21] L. Ljung (1977) Analysis of recursive stochastic algorithms, IEEE Trans Autom. Control,AC-22:551-575.

[22] D. Monderer and L. S. Shapley, (1996) Fictitious play property for games with identical interests, J. Economic Theory, 68, 258-265. 
[23] J. Robinson, (1951) An iterative method of solving a game, Annals of Mathematics, 54, 296-301.

[24] S. Smale, (1980) The prisoner's dilemma and dynamical systems associated to non-cooperative games, Econometrica, 48, 1617-1633.

[25] S. Sorin, (2002) A First Course on Zero-Sum Repeated Games, Springer . 